POS PROCEEDINGS

\title{
Magnus and Dyson Series for Master Integrals
}

\author{
Mario Argeri \\ Dipartimento di Scienze e Innovazione Tecnologica, Università del Piemonte Orientale, Viale \\ Teresa Michel 11, I-15121 Alessandria, Italy \\ E-mail: mario.argeri@mfn.unipmn.it
}

\section{Stefano Di Vita}

Max-Planck-Institut für Physik, Föhringer Ring 6, D-80805 München, Germany; E-mail:

divita@mpp.mpg • de

\section{Pierpaolo Mastrolia*}

Max-Planck-Institut für Physik, Föhringer Ring 6, D-80805 München, Germany;

Dipartimento di Fisica e Astronomia, Università di Padova, and INFN Sezione di Padova, via Marzolo 8, 35131 Padova, Italy

E-mail: pierpaolo.mastrolia@cern.ch

\section{Edoardo Mirabella}

Max-Planck-Institut für Physik, Föhringer Ring 6, D-80805 München, Germany; E-mail: mirabellempp.mpg • de

\section{Johannes Schlenk}

Max-Planck-Institut für Physik, Föhringer Ring 6, D-80805 München, Germany; E-mail: jschlenk@mpp.mpg.de

\section{Ulrich Schubert}

Max-Planck-Institut für Physik, Föhringer Ring 6, D-80805 München, Germany; E-mail: schubert ampp.mpg • de

\section{Lorenzo Tancredi}

Physik-Institut, Universität Zürich, Wintherturerstrasse 190, CH-8057 Zürich, Switzerland E-mail: tancredi@physik.uzh.ch

The Magnus Exponential can be employed to solve the system of differential equations obeyed by Feynman integrals in dimensional regularization. We discuss the basic ideas behind the use of Magnus series expansion, and how the solution naturally arises in terms of repeated integrals that are equivalent to the coefficients of a Dyson series. Finally, we show its application to the evaluation of planar and non-planar two-loop QED vertex diagrams for massive fermions, and to non-planar two-loop integrals contributing to $2 \rightarrow 2$ scattering of massless particles.

Loops and Legs in Quantum Field Theory - LL 2014,

27 April - 2 May 2014

Weimar, Germany

\footnotetext{
*Speaker.
} 


\section{Introduction}

The method of differential equations [1-3] (see also Ref. [4,5]), is one of the most effective techniques for computing dimensionally regulated multi-loop integrals. Within the continuous dimensional regularization scheme, Feynman integrals fulfill identities that fall in the catheogry of the general class of integration-by-parts relations [6,7]. Such relations can be exploited in order to identify a set of independent integrals, dubbed master integrals (MI's), that can be used as a basis of functions for the virtual contributions to scattering amplitudes.

The MI's are functions of the kinematic invariants constructed from the external momenta and of the masses of the (internal and external) particles. Remarkably, the aforementioned relations imply that the MI's obey linear systems of first-order differential equations (DE's) in the kinematic invariants, which can be used for the determination of their actual expression. The solution of the system, namely the MI's, is finally determined by imposing the boundary conditions at special values of the kinematic variables, properly chosen either in correspondence of configurations that reduce the MI's to simpler integrals or in correspondence of pseudo-thresholds. In this latter case, the boundary conditions are obtained by imposing the regularity of the MI's around unphysical singularities, ruling out divergent behavior of the general solution of the systems.

For any given scattering process the set of MI's is not unique, and, in practice, their choice is rather arbitrary. Usually MI's are identified after applying the Laporta reduction algorithm [8]. Afterward, convenient manipulations of the basis of MI's may be performed. Proper choices of MI's can simplify the form of the systems of differential equations, hence, of their solution, although general criteria for determining such optimal sets are not available. In Ref. [9], Henn proposes to solve the systems of DE's for MI's with algebraic methods, by observing that with a good choice of MI's the system of DE's can be cast in a form - which we define canonical - where the dependence on the dimensional parameter $\varepsilon=(4-d) / 2$ is factorized from the kinematic. The integration of a system in canonical form trivializes and the analytic properties of its general solution are manifestly inherited from the matrix associated to the system, which is the kernel of the representation of the solutions in terms of repeated integrations.

In Ref. [10], we suggest a convenient form for the initial system of MI's, and we propose an algorithm to find the transformation matrix yielding to a canonical system. In particular, we choose a set of MI's obeying a systems of DE's which has a linear $\varepsilon$-dependence, and we find a transformation which absorbs the $\mathscr{O}\left(\varepsilon^{0}\right)$ term and leads to a new system of DE's where the $\varepsilon$ dependence is factorized. This transformation, as well as the integration of the canonical system, are obtained by using Magnus and Dyson's series expansions [11-13].

We apply our algorithm to compute the MI's of the two-loop vertex diagrams contributing to the massive fermion form-factors in QED [14,15] and the MI's of the non-planar two-loop diagrams contributing to the $2 \rightarrow 2$ scattering of massless particles $[17,18]$. The set of MI's for the two-loop QED vertices hereby presented constitutes a transcendentally-homogeneous subset for tackling the analytic calculation of the still unknown non-planar two-loop box diagrams contributing to massive Bhabha scattering in QED. It may enter as well in more general classes of scattering processes involving massive particles.

We used the computer code REDUZE2 [19] for the generation of the systems of differential equations. 


\section{Magnus series expansion}

Consider a generic linear matrix differential equation [13]

$$
\partial_{x} Y(x)=A(x) Y(x), \quad Y\left(x_{0}\right)=Y_{0} .
$$

If $A(x)$ commutes with its integral $\int_{x_{0}}^{x} d \tau A(\tau), e . g$. in the scalar case, the solution can be written as

$$
Y(x)=e^{\int_{x_{0}}^{x} d \tau A(\tau)} Y_{0} .
$$

In the general non-commutative case, one can use the Magnus theorem [11] to write the solution as,

$$
Y(x)=e^{\Omega\left(x, x_{0}\right)} Y\left(x_{0}\right) \equiv e^{\Omega(x)} Y_{0}
$$

where $\Omega(x)$ is written as a series expansion, called Magnus expansion,

$$
\Omega(x)=\sum_{n=1}^{\infty} \Omega_{n}(x) .
$$

The first three terms of the expansion (2.4) read as follows:

$$
\begin{aligned}
& \Omega_{1}(x)=\int_{x_{0}}^{x} d \tau_{1} A\left(\tau_{1}\right) \\
& \Omega_{2}(x)=\frac{1}{2} \int_{x_{0}}^{x} d \tau_{1} \int_{x_{0}}^{\tau_{1}} d \tau_{2}\left[A\left(\tau_{1}\right), A\left(\tau_{2}\right)\right], \\
& \Omega_{3}(x)=\frac{1}{6} \int_{x_{0}}^{t} d \tau_{1} \int_{x_{0}}^{\tau_{1}} d \tau_{2} \int_{x_{0}}^{\tau_{2}} d \tau_{3}\left[A\left(\tau_{1}\right),\left[A\left(\tau_{2}\right), A\left(\tau_{3}\right)\right]\right]+\left[A\left(\tau_{3}\right),\left[A\left(\tau_{2}\right), A\left(\tau_{1}\right)\right]\right] .
\end{aligned}
$$

We remark that if $A$ and its integral commute, the series (2.4) is truncated at the first order, $\Omega=\Omega_{1}$, and we recover the solution (2.2). As a notational aside, in the following we will use the symbol $\Omega[A](x)$ to denote the Magnus expansion obtained using $A$ as kernel.

\subsection{Magnus and Dyson series expansion}

Magnus series is related to the Dyson series [13], and their connection can be obtained starting from the Dyson expansion of the solution of the system (2.1),

$$
Y(x)=Y_{0}+\sum_{n=1}^{\infty} Y_{n}(x), \quad Y_{n}(x) \equiv \int_{x_{0}}^{x} d \tau_{1} \ldots \int_{x_{0}}^{\tau_{n-1}} d \tau_{n} A\left(\tau_{1}\right) A\left(\tau_{2}\right) \cdots A\left(\tau_{n}\right)
$$

in terms of the time-ordered integrals $Y_{n}$. Comparing Eq. (2.3) and (2.6) we have

$$
\sum_{j=1}^{\infty} \Omega_{j}(x)=\log \left(Y_{0}+\sum_{n=1}^{\infty} Y_{n}(x)\right) .
$$

In the following, we will use both Magnus and Dyson series. The former allows us to easily demonstrate how a system of DE's, whose matrix is linear in $\varepsilon$, can be cast in the canonical form. The latter can be more conveniently used for the explicit representation of the solution. 


\section{Differential equations for Master Integrals}

We consider a linear system of first order differential equations

$$
\partial_{x} f(\varepsilon, x)=A(\varepsilon, x) f(\varepsilon, x),
$$

where $f$ is a vector of MI's, while $x$ is a variable depending on kinematic invariants and masses. We suppose that $A$ depends linearly on $\varepsilon$,

$$
A(\varepsilon, x)=A_{0}(x)+\varepsilon A_{1}(x),
$$

and we change the basis of MI's via the Magnus series obtained by using $A_{0}$ as kernel,

$$
f(\varepsilon, x)=B_{0}(x) g(\varepsilon, x), \quad B_{0}(x) \equiv e^{\Omega\left[A_{0}\right]\left(x, x_{0}\right)} .
$$

Because of Magnus Theorem, $B_{0}$ obeys the equation,

$$
\partial_{x} B_{0}(x)=A_{0}(x) B_{0}(x),
$$

which implies that the new basis $g$ of MI's fulfills a system of differential equations in the canonical factorized form,

$$
\partial_{x} g(\varepsilon, x)=\varepsilon \hat{A}_{1}(x) g(\varepsilon, x) .
$$

The matrix $\hat{A}_{1}$ is related to $A_{1}$ through,

$$
\hat{A}_{1}(x)=B_{0}^{-1}(x) A_{1}(x) B_{0}(x),
$$

and does not depend on $\varepsilon$. The solution of Eq. (3.5) can be found by using Magnus theorem with $\varepsilon \hat{A}_{1}$ as kernel

$$
g(\varepsilon, x)=B_{1}(\varepsilon, x) g_{0}(\varepsilon), \quad B_{1}(\varepsilon, x)=e^{\Omega\left[\varepsilon \hat{A}_{1}\right]\left(x, x_{0}\right)},
$$

where the vector $g_{0}$ corresponds to the boundary values of the MI's. Therefore, the solution of the original system Eq. (3.1) finally reads,

$$
f(\varepsilon, x)=B_{0}(x) B_{1}(\varepsilon, x) g_{0}(\varepsilon) .
$$

It is worth to notice that $\Omega\left[\varepsilon \hat{A}_{1}\right]$ in Eq. (3.7) depends on $\varepsilon$, while $\Omega\left[A_{0}\right]$ in Eq. (3.3) does not.

The matrix $B_{0}$, implementing the transformation from the linear to the canonical form, is simply given as the product of two matrix exponentials. Indeed one can split $A_{0}$ into a diagonal term, $D_{0}$, and a matrix with vanishing diagonal entries $N_{0}$,

$$
A_{0}(x)=D_{0}(x)+N_{0}(x)
$$

The transformation $B$ is then obtained by the composition of two transformations

$$
B(x)=e^{\Omega\left[D_{0}\right]\left(x, x_{0}\right)} e^{\Omega\left[\hat{N}_{0}\right]\left(x, x_{0}\right)}=e^{\int_{x_{0}}^{x} d \tau D_{0}(\tau)} e^{\Omega\left[\hat{N}_{0}\right]\left(x, x_{0}\right)},
$$




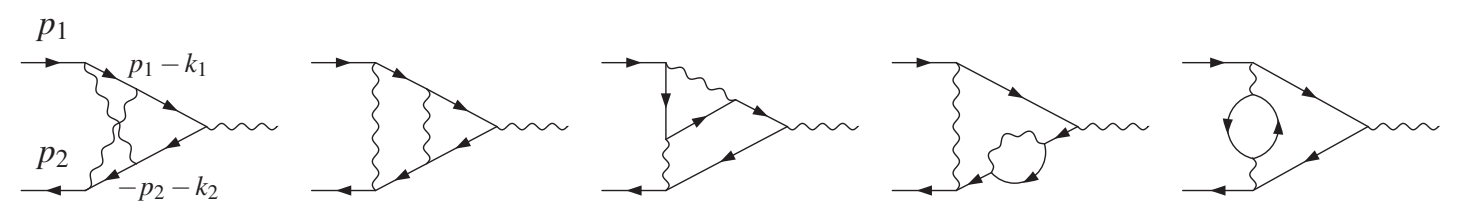

Figure 1: Selection of Feynman diagrams entering the correction of the QED vertex at two loops. The internal momenta in the first diagram are oriented according to the fermion flow, while the external momenta are incoming.

where $\hat{N}_{0}$ is given by

$$
\hat{N}_{0}(x)=e^{-\int_{x_{0}}^{x} d \tau D_{0}(\tau)} N_{0}(x) e^{\int_{x_{0}}^{x} d \tau D_{0}(\tau)}
$$

In the last step of Eq. (3.10) we have used the commutativity of the diagonal matrix $D_{0}$ with its own integral. We also remark that the decomposition in Eq.(3.9) accelerates the convergence of the Magnus series.

In the examples hereby discussed it was possible, by trials and errors, to find set of MI's obeying a system of DE's linear in $\varepsilon$. Moreover in these cases one finds that $\Omega\left[\hat{N}_{0}\right]$ contains just the first term of the series, except for the non-planar box, where also the second order is non vanishing.

\section{Two-Loop QED Vertices}

A set of MI's entering the electron form factor at two loop in QED [15] were computed in Ref. [14], for arbitrary kinematic and finite electron mass. The contributing diagrams are depicted in Fig. 1 and depend on $s=\left(p_{1}+p_{2}\right)^{2}$ and $p_{1}^{2}=p_{2}^{2}=m^{2}$. In this example we choose an alternative set of MI's,

$$
\begin{aligned}
& f_{1}=\varepsilon^{2} \mathscr{T}_{1}, \quad f_{2}=\varepsilon^{2} \mathscr{T}_{2}, \quad f_{3}=\varepsilon^{2} \mathscr{T}_{3}, \quad f_{4}=\varepsilon^{2} \mathscr{T}_{4}, \quad f_{5}=\varepsilon^{2} \mathscr{T}_{5}, \\
& f_{6}=\varepsilon^{2} \mathscr{T}_{6}, \quad f_{7}=\varepsilon^{2} \mathscr{T}_{7}, \quad f_{8}=\varepsilon^{3} \mathscr{T}_{8}, \quad f_{9}=\varepsilon^{3} \mathscr{T}_{9}, \quad f_{10}=\varepsilon^{2} \mathscr{T}_{10}, \\
& f_{11}=\varepsilon^{3} \mathscr{T}_{11}, \quad f_{12}=\varepsilon^{3} \mathscr{T}_{12}, \quad f_{13}=\varepsilon^{2} \mathscr{T}_{13}, \quad f_{14}=\varepsilon^{3} \mathscr{T}_{14}, \quad f_{15}=\varepsilon^{4} \mathscr{T}_{15}, \\
& f_{16}=\varepsilon^{4} \mathscr{T}_{16}, \quad f_{17}=\varepsilon^{4} \mathscr{T}_{17},
\end{aligned}
$$

where the integrals $\mathscr{T}_{i}$ are collected in Fig. 2. The system of differential equations for $f$, in the auxiliary variable $x$, defined through $s=-\frac{m^{2}(1-x)^{2}}{x}$ is linear in $\varepsilon$. Therefore after applying Magnus 


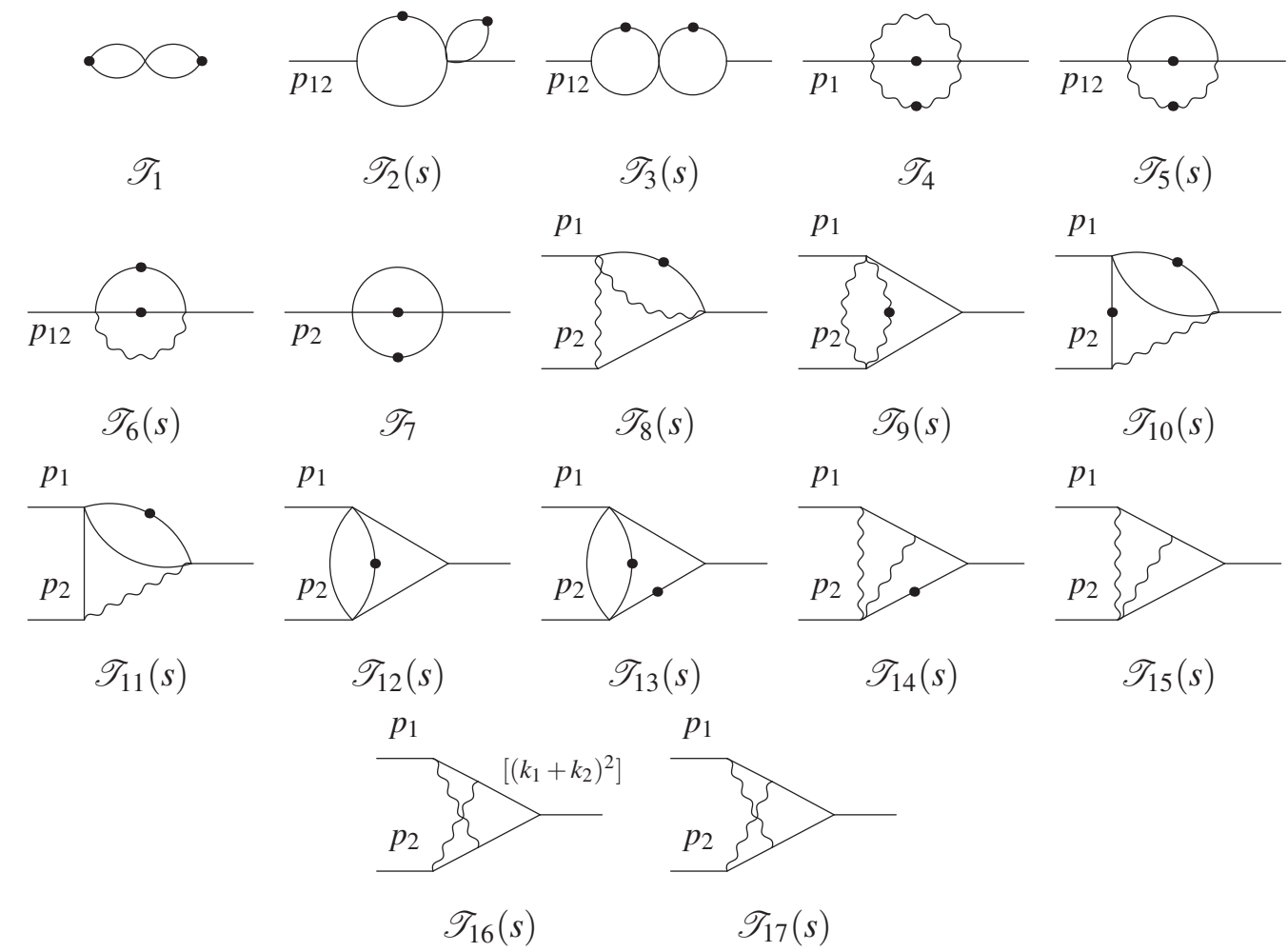

Figure 2: MI's for the two-loop corrections of the QED vertex. All the external momenta depicted are incoming. In the integral $\mathscr{T}_{16}$ the loop momenta $k_{1}, k_{2}$ are fixed according to the first diagram of Fig. 1 and a term $\left(k_{1}+k_{2}\right)^{2}$ has to be included in the numerator of the integrand.

rotation, the canonical basis $g$ is found,

$$
\begin{aligned}
& g_{1}=f_{1}, \\
& g_{2}=\lambda_{1} f_{2}, \\
& g_{3}=(-s) \lambda_{2} f_{3}, \\
& g_{4}=m^{2} f_{4} \text {, } \\
& g_{5}=\lambda_{1}\left(f_{5}+\frac{f_{6}}{2}\right)-\frac{s}{2} f_{6}, \quad g_{6}=(-s) f_{6}, \\
& g_{7}=m^{2} f_{7}, \\
& g_{8}=\lambda_{1} f_{8}, \\
& g_{9}=\lambda_{1} f_{9}, \\
& g_{10}=\lambda_{3}\left(2 f_{5}+f_{6}\right)+m^{2} \lambda_{2} f_{10}, \\
& g_{11}=\lambda_{1} f_{11} \text {, } \\
& g_{12}=\lambda_{1} f_{12} \text {, } \\
& g_{13}=\left(3 m^{2}-\frac{3 s}{2}\right) f_{7}-s \lambda_{2} f_{13}, \\
& g_{14}=(-s) \lambda_{2} f_{14}, \\
& g_{15}=\lambda_{1} f_{15}, \\
& g_{16}=\lambda_{1} f_{16}, \\
& g_{17}=(-s) \lambda_{2} f_{17},
\end{aligned}
$$

where $\lambda_{1}=\sqrt{-s} \sqrt{4 m^{2}-s}, \lambda_{2}=\left(4 m^{2}-s\right), \lambda_{3}=\frac{\lambda_{1}+\lambda_{2}}{4}$. The new basis of MI's obeys a canonical system of DE,

$$
\partial_{x} g(\varepsilon, x)=\varepsilon \hat{A}_{1}(x) g(\varepsilon, x), \quad \hat{A}_{1}(x)=\frac{M_{1}}{x}+\frac{M_{2}}{1+x}+\frac{M_{3}}{1-x},
$$

where $M_{i},(i=1,2,3)$ are sparse matrix with rational numbers.

The solution of the system can be expressed as Dyson series, as well as Magnus series, in terms of one-dimensional Harmonic Polylogarithms (HPL's) [20]. The requirements that the MI's 


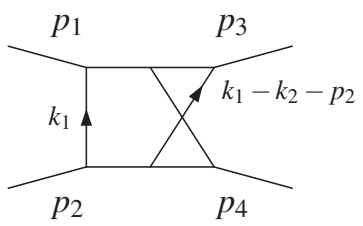

Figure 3: Non-planar two-loop diagram with massless internal propagators, and massless external particles. The internal momenta shown in the diagram are oriented according to the arrows. All the external momenta are incoming.

are real-valued in the Euclidean region and regular in $x=1(s=0)$, or simply the matching against the known integrals at $x=1$, fix all but three boundary constants, corresponding to the constant MI's $g_{1}, g_{4}$ and $g_{7}$ (that do not depend on $x$ ). The integrals $g_{1}$ and $g_{4}$ can be easily computed by direct integration, while $g_{7}$ can be determined from the results of Ref. [16]. The matrices $M_{i},(i=1,2,3)$ and the expressions of the transcendentally uniform MI's $g_{i},(i=1, \ldots, 17)$ can be found in Ref. [10].

\section{Two-Loop non-planar Box}

The evaluation of the two-loop non-planar box diagram in Fig. 3, contributing to the $2 \rightarrow 2$ scattering among massless particles, has already been considered in the literature [17,18]. Recently, for its planar partner, a set of MI's with homogenous transcendentality was presented in Ref. [9]. In this section, we compute the additional MI's required for determining the non-planar contribution, having expressions with manifest homogenous transcendentality as well. The integrals, in this case, are functions of the invariants $s=\left(p_{1}+p_{2}\right)^{2}, t=\left(p_{1}+p_{3}\right)^{2}$, and $u=\left(p_{2}+p_{3}\right)^{2}$, with $p_{i}^{2}=0$, and $s+t+u=0$. We begin with the following initial choice of MI's,

$$
\begin{array}{ccc}
f_{1}=\varepsilon^{2} s \mathscr{T}_{a}(s), & f_{2}=-\varepsilon^{2} u \mathscr{T}_{a}(u), & f_{3}=\varepsilon^{2} t \mathscr{T}_{a}(t), \\
f_{4}=\varepsilon^{3} s \mathscr{T}_{b}(s), & f_{5}=\varepsilon^{3} s t \mathscr{T}_{c}(s, t) & f_{6}=-\varepsilon^{3} s u \mathscr{T}_{c}(s, u), \\
f_{7}=-\varepsilon^{4} u \mathscr{T}_{d}(s, t), & f_{8}=\varepsilon^{4} s \mathscr{T}_{d}(u, t), & f_{9}=\varepsilon^{4} t \mathscr{T}_{d}(s, u), \\
f_{10}=\varepsilon^{4} s^{2} \mathscr{T}_{e}(s), & \\
f_{11}=-\varepsilon^{4} s t u \mathscr{T}_{f}(s, t)+\frac{1}{1+4 \varepsilon}\left[\frac{3 \varepsilon^{2}}{4 s}\left(s^{2} \mathscr{T}_{a}(s)+u^{2} \mathscr{T}_{a}(u)+t^{2} \mathscr{T}_{a}(t)\right)\right. \\
\left.-\frac{3 \varepsilon^{4}}{s}\left(u^{2} \mathscr{T}_{d}(s, t)+s^{2} \mathscr{T}_{d}(u, t)+t^{2} \mathscr{T}_{d}(s, u)\right)\right] \\
f_{12}=\varepsilon^{4} s t \mathscr{T}_{g}(s, t)-\frac{1}{2(1+4 \varepsilon)}\left[\frac{3 \varepsilon^{2}}{4 u}\left(s^{2} \mathscr{T}_{a}(s)+u^{2} \mathscr{T}_{a}(u)+t^{2} \mathscr{T}_{a}(t)\right)\right. \\
\left.-\frac{3 \varepsilon^{4}}{u}\left(u^{2} \mathscr{T}_{d}(s, t)+s^{2} \mathscr{T}_{d}(u, t)+t^{2} \mathscr{T}_{d}(s, u)\right)\right]
\end{array}
$$

where the integrals $\mathscr{T}$ 's correspond to the diagrams in Fig. 4 . We notice, that $f_{i}$ with $(i=1, \ldots, 9)$ are common to the two-loop planar box diagram [9]. The set $f$ of MI's obeys a system of differential 


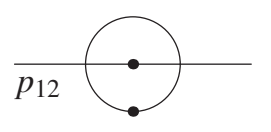

$\mathscr{T}_{a}(s)$

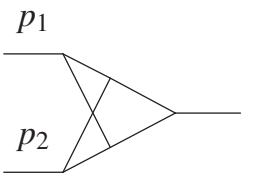

$\mathscr{T}_{e}(s)$

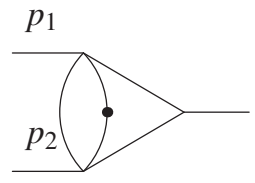

$\mathscr{T}_{b}(s)$

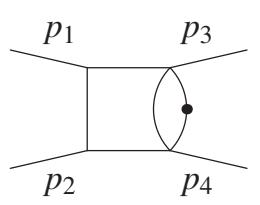

$\mathscr{T}_{c}(s, t)$

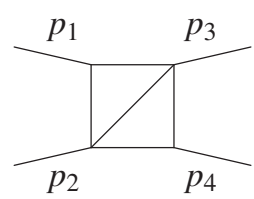

$\mathscr{T}_{d}(s, t)$

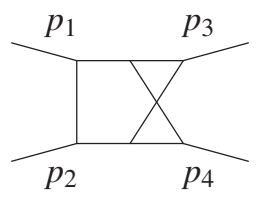

$\mathscr{T}_{f}(s, t)$

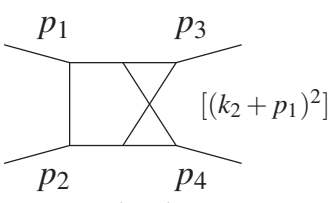

$\mathscr{T}_{g}(s, t)$

Figure 4: MI's for the two-loop diagram in Fig. 3. All the external momenta depicted are incoming. In the last integral the loop momenta have to be fixed according to Fig. 3 and a term $\left(k_{2}+p_{1}\right)^{2}$ enters the numerator of its integrand.

equations the variable $x$, defined as, $x=-\frac{t}{s}$, which is linear in $\varepsilon$.

According to the procedure in Section 3, we can build the matrix $B_{0}(x)$ ruling the change of basis $f(\varepsilon, x)=B_{0}(x) g(\varepsilon, x)$, so that the new MI's,

$$
\begin{aligned}
g_{i} & =f_{i}, \quad 1 \leq i \leq 10, \\
g_{11} & =\frac{s}{8 t u}\left[t\left(9 f_{1}-6 f_{2}-3 f_{3}-8 f_{6}-12 f_{8}+36 f_{9}\right)\right. \\
& \left.+u\left(-15 f_{1}-3 f_{2}-12 f_{3}-16 f_{5}+60 f_{7}+12 f_{8}+8 f_{11}-8 f_{12}\right)\right], \\
g_{12} & =\frac{s}{8 u}\left(9 f_{1}-6 f_{2}-3 f_{3}-8 f_{6}-12 f_{8}+36 f_{9}\right)+f_{12},
\end{aligned}
$$

obey the canonical system,

$$
\partial_{x} g(\varepsilon, x)=\varepsilon \hat{A}_{1}(x) g(\varepsilon, x), \quad \hat{A}(x)=\frac{M_{1}}{x}+\frac{M_{2}}{1-x},
$$

where $M_{i},(i=1,2)$ are sparse matrix with rational numbers.

The solution of the system can be expressed as Dyson series, as well as Magnus series, in terms of one-dimensional HPL's [20]. As long as the planar sub topologies are concerned, one can fix the boundary conditions using the regularity properties of the integrals in some special kinematical points. On the other hand, the analyticity structure of the crossed box is more complicated, since it involves at the same time cuts in all three Mandelstam variables $s, t, u$. Nevertheless, in this particular case, the boundaries can be fixed by direct comparison with the results presented in $[17,18]$. The matrices $M_{i},(i=1,2)$ and the expressions of the transcendentally uniform MI's $g_{i},(i=1, \ldots, 12)$ can be found in Ref. [10].

\section{Conclusions}

In this contribution, we elaborated on the method of differential equations for Feynman integrals within the $D$-dimensional regularization scheme. We exploited the the freedom of choosing a suitable basis of MI's to analyze the paradigmatic case of systems of differential equations whose 
matrix is linear in the dimensional parameter, $\varepsilon=(4-D) / 2$. We show that these systems can admit a canonical form, where the dependence on $\varepsilon$ is factorized from the kinematic variables, as recently suggested by Henn.

We used Magnus series to obtain the matrix implementing the transformation from the linear to the canonical form. The solution of the canonical system is obtained by using either Dyson series or Magnus series. Both series requires multiple integrations which allows one to naturally express the MI's in terms of (generalized) polylogarithms.

We recalled the results presented in Ref. [10], where the method was applied to the two-loop electron form factors in QED and the the two-loop contributions to the massless $2 \rightarrow 2$ scattering.

We would like to conclude this contribution with a series of comments and open questions.

Further investigation is required for clarifying whether the possibility of choosing a set of master integrals obeying a system that is linear in $\varepsilon$ is a general feature or just accidental, and, eventually, how to find it in a systematic way. In this respect, we think that it might be worth to consider systems of differential equations for master integrals that are not necessarily all defined at the same value of space-time dimensions, because regularity in the $\varepsilon \rightarrow 0$ limit and uniform transcendentality may be a property of shifted-dimension master integrals, and the shifting amount could depend on the topology of the diagrams.

Moreover, even in the case a set of MI's obeying a system of differential equations linear in $\varepsilon$ is found, the convergence of the Magnus series, needed for finding a finite matrix that implements the transformation to the canonical form, is not guaranted a priori.

Therefore, we can turn the arrow of the implications around, and conjecture that the existence of a canonical set of MI's that can be expressed in terms of (generalised) polylogarithms of uniform transcendentality and obeying a canonical system of differential equations implies the existence of a (finite) Magnus exponential matrix that rules the transformation of the canonical basis to a basis obeying a system that is linear in $\varepsilon$.

Nevertheless, it is also known that (generalised) polylogarithms do not exhaust the set of functions appearing in the evaluation of Feynman integrals, where also elliptic functions do arise. What does happen in these cases? Does a $\varepsilon$-linear system exist? Can one find a converging Magnus exponential matrix? Can the convergence of the Magnus exponential capture the ellipticity or the polylogarithmicity of the MI's?

Answering to these questions requires definitely further studies and more applications to cases of increasing complexity, which we plan for the near future.

\section{Acknowledgments}

P.M. whishes to thank Ettore Remiddi for enjoyful and stimultating discussions that took place during the workshop, and kept on going since then. The work of P.M. and U.S. is supported by the Alexander von Humboldt Foundation, in the framework of the Sofja Kovalevskaja Award 2010, endowed by the German Federal Ministry of Education and Research. 


\section{References}

[1] A. V. Kotikov, Differential equations method: New technique for massive Feynman diagrams calculation, Phys. Lett. B 254 (1991) 158.

[2] E. Remiddi, Differential equations for Feynman graph amplitudes, Nuovo Cim. A 110 (1997) 1435 [hep-th/9711188].

[3] T. Gehrmann and E. Remiddi, Differential equations for two loop four point functions, Nucl. Phys. B 580 (2000) 485 [hep-ph/9912329].

[4] M. Argeri and P. Mastrolia, Feynman Diagrams and Differential Equations, Int. J. Mod. Phys. A 22 (2007) 4375 [arXiv:0707.4037 [hep-ph]].

[5] V. A. Smirnov, Analytic tools for Feynman integrals, Springer Tracts Mod. Phys. 250 (2012) 1.

[6] K. G. Chetyrkin, A. L. Kataev and F. V. Tkachov, New Approach to Evaluation of Multiloop Feynman Integrals: The Gegenbauer Polynomial x Space Technique, Nucl. Phys. B 174 (1980) 345.

[7] F. V. Tkachov, A Theorem on Analytical Calculability of Four Loop Renormalization Group Functions, Phys. Lett. B 100 (1981) 65.

[8] S. Laporta, High precision calculation of multiloop Feynman integrals by difference equations, Int. J. Mod. Phys. A 15 (2000) 5087 [hep-ph/0102033].

[9] J. M. Henn, Multiloop integrals in dimensional regularization made simple, Phys. Rev. Lett. 110 (2013) 25, 251601 [arXiv:1304.1806 [hep-th]].

[10] M. Argeri, S. Di Vita, P. Mastrolia, E. Mirabella, J. Schlenk, U. Schubert and L. Tancredi, Magnus and Dyson Series for Master Integrals, JHEP 1403 (2014) 082 [arXiv:1401.2979 [hep-ph]].

[11] W. Magnus, On the exponential solution of differential equations for a linear operator, Comm. Pure and Appl. Math. VII (1954).

[12] F. J. Dyson, The Radiation theories of Tomonaga, Schwinger, and Feynman, Phys. Rev. 75 (1949) 486.

[13] S. Blanes, F. Casas, J. A. Oteo, and J. Ros, The magnus expansion and some of its applications, Physics Reports 470 (2009) [ arXiv:0810.5488 [math-ph]].

[14] R. Bonciani, P. Mastrolia and E. Remiddi, Vertex diagrams for the QED form-factors at the two loop level, Nucl. Phys. B 661 (2003) 289 [Erratum-ibid. B 702 (2004) 359] [hep-ph/0301170].

[15] R. Bonciani, P. Mastrolia and E. Remiddi, QED vertex form-factors at two loops, Nucl. Phys. B 676 (2004) 399 [hep-ph/0307295].

[16] M. Argeri, P. Mastrolia and E. Remiddi, The Analytic value of the sunrise selfmass with two equal masses and the external invariant equal to the third squared mass, Nucl. Phys. B 631 (2002) 388 [hep-ph/0202123].

[17] J. B. Tausk, Nonplanar massless two loop Feynman diagrams with four on-shell legs, Phys. Lett. B 469 (1999) 225 [hep-ph/9909506].

[18] C. Anastasiou, T. Gehrmann, C. Oleari, E. Remiddi and J. B. Tausk, The Tensor reduction and master integrals of the two loop massless crossed box with lightlike legs, Nucl. Phys. B 580 (2000) 577 [hep-ph/0003261].

[19] A. von Manteuffel and C. Studerus, Reduze 2 - Distributed Feynman Integral Reduction, arXiv:1201.4330 [hep-ph].

[20] E. Remiddi and J. A. M. Vermaseren, Harmonic polylogarithms, Int. J. Mod. Phys. A 15 (2000) 725 [hep-ph/9905237]. 